\title{
NEW UPPER AND LOWER BOUNDS LINE OF SIGHT PATH LOSS MODELS FOR MOBILE PROPAGATION IN BUILDINGS
}

\author{
Supachai Phaiboon* \\ Electrical Engineering Department, Faculty of Engineering, \\ Mahidol University Pisit, Thailand \\ Phokharatkul \\ Computer Engineering Department, Faculty of Engineering, \\ Mahidol University, Thailand

\section{Suripon Somkuarnpanit} \\ Electronic Department, Faculty of Engineering, \\ King Mongkut's Institute of Technology Ladkrabang, Thailand
}

Received 16 March 2006

\begin{abstract}
This paper proposes a method to predict line-of-sight (LOS) path loss in buildings. We performed measurements in two different type of buildings at a frequency of $1.8 \mathrm{GHz}$ and propose new upper and lower bounds path loss models which depend on max and min values of sample path loss data. This makes our models limit path loss within the boundary lines. The models include time-variant effects such as people moving and cars in parking areas with their influence on wave propagation that is very high. The results have shown that the proposed models will be useful for the system and cell design of indoor wireless communication systems.
\end{abstract}

Keywords: Indoor LOS path loss, upper and lower bounds, time-variant effects

\section{INTRODUCTION}

Indoor wireless communication is widely used in different type of buildings therefore it needs suitable network planning to provide the best service. This makes it necessary to have a way to predict the propagation in indoor environments in order to determine the best location of the base stations to provide an efficient service. Another objective in the network designs is the limiting of the received signal level in order to comply with public health regulations.

For indoor communication system design, the line-of-sight (LOS) path loss characteristics have to be clarified because the transmitter is generally installed in section of a corridor. Therefore, a path loss model for LOS is still needed. Previously, there were three different

${ }^{*}$ Corresponding author e-mail: egspb@mahidol.ac.th 
approaches for the prediction of the field strength, both LOS and non line-of-sight (NLOS), inside buildings. On the one hand there are empirical models, based on the regression of measurement data $[1,2]$. There are also deterministic models like ray tracing [3 - 5]. On the other hand, there are semi-deterministic models, based on the regression of measurement data and/or some of the uniform theory of diffraction (UTD) [6]. These models do not include timevariant effects such as people moving, cars in parking areas and door or window opening. Their influence on wave propagation are very high, because dimensions of windows, doors, people or cars are nearly equal to dimensions of time-invariant walls and columns, while in outdoor environments, time-variant effects are often negligible because their dimensions are very small compared with dimensions of time-invariant buildings.

In deterministic models, it is not possible to include these time-variant effects in the data base and scattering is ignored. Although the authors [7] have shown the effect of the human body in indoor environments, but they have not considered the nature of people in the building nor shown the building data base in details.

To solve this problem, we propose a new upper and lower bounds LOS model that takes into consideration the time-variant effects, including breakpoint distance. The spread of upper- and lower-bounds depend on max and min value of a sample path loss data, therefore they provide accurate prediction while they still maintained least square error. Although upper-and lowerbound estimations have been performed for outdoor communication in UHF band [8] and microwave band [9], they can not be applied for indoor communication because of the different environment. We found that the breakpoint distance depends on ceiling height and is independent from corridor width. Because there are only three dominant reflections, one from the direct path, two from the floor, and another from the ceiling, the reflections from side walls within the studied buildings suffer from numerous losses due to penetration through glass, porous materials, etc., before reflection.

\section{MEASUREMENT PROCEDURE AND LOCATION}

\subsection{Measurement procedure}

The equipment for propagation measurement consisted of a fixed transmitter and a narrow- band $(20 \mathrm{KHz})$ portable spectrum interfaced with a microcomputer at a frequency of $1.8 \mathrm{GHz}$. The fixed transmitter consisted of a network analyzer (with $18 \mathrm{dBm}$ power output) and $\lambda / 4$ omnidirectional antenna with $10 \times 10 \mathrm{~cm}^{2}$ ground plane $(2.2 \mathrm{dBi}$ gain). We also used the same type antenna for signal strength measurement via recorder as shown in Fig. 1.

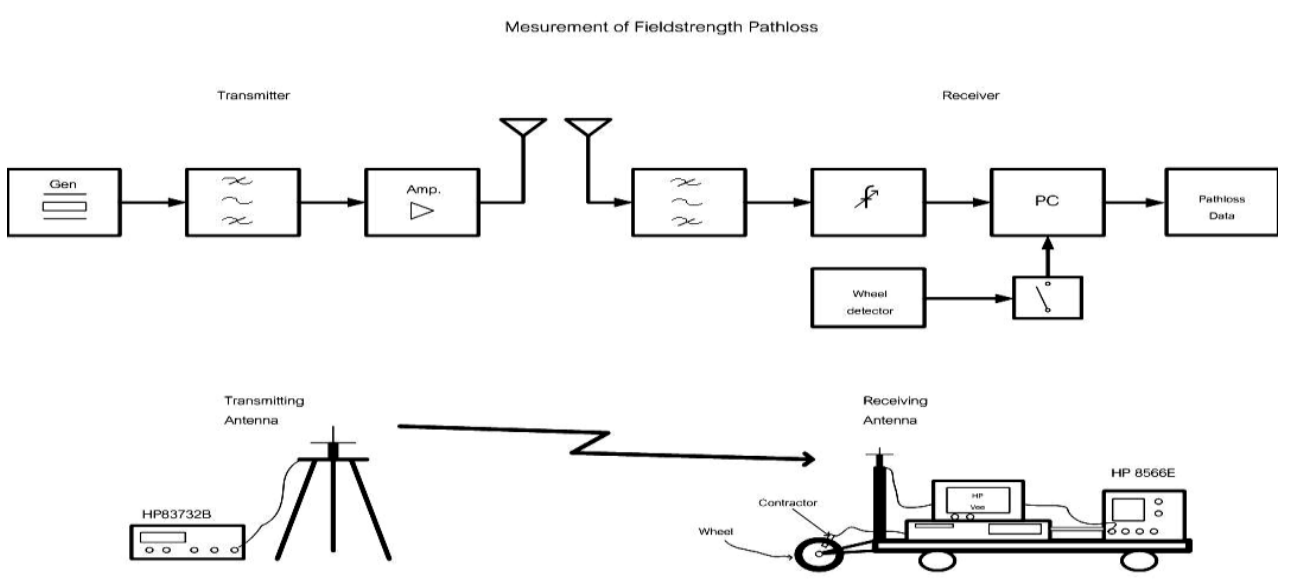

Fig. 1: Measurement system.

408 
To receive propagation data for modelling, the samples of the actual field at a frequency of 1800 $\mathrm{MHz}$ in the building were acquired by moving the mobile unit along LOS corridors in the building areas. Since the standing waves repeat every $0.5 \lambda$, we took the sample data every distance of $0.25 \lambda$. The velocity of the mobile is about $8.3 \mathrm{~cm} / \mathrm{s}$, then the effective sampling rate is about 2 samples/s at the frequency of $1.8 \mathrm{GHz}$.

To characterize the time-variant objects along the LOS way, we selected days and times with high traffic in the buildings for recording. That is at midday for a school building and a Saturday afternoon for a Future park building.

\subsection{Measurement location}

The stationary transmitter was placed at the end of a corridor of the building to determine the effects of wave propagation. For measurement locations, the mobile receiver thoroughly moved the building at transmitter-receiver (T-R) separations in LOS corridors. For modeling, two different buildings were considered as follows:

\subsubsection{Concrete school building}

The concrete building of the Faculty of Engineering, Mahidol University was completed in 1993. It consists of five floors with dimensions of $100 \times 270 \mathrm{~m}^{2}$ as shown in Fig. 2. The construction of the building is of concrete block, plaster board, and mirror walls, the floor to ceiling height is $3.5 \mathrm{~m}$ with a light plasterboard suspended ceiling containing air conditioning and service ducts $2.6 \mathrm{~m}$ above the floor. The material of walls in corridor are $60 \%$ of glass and $40 \%$ of concrete block. The building capacity is an average 490 people per floor.

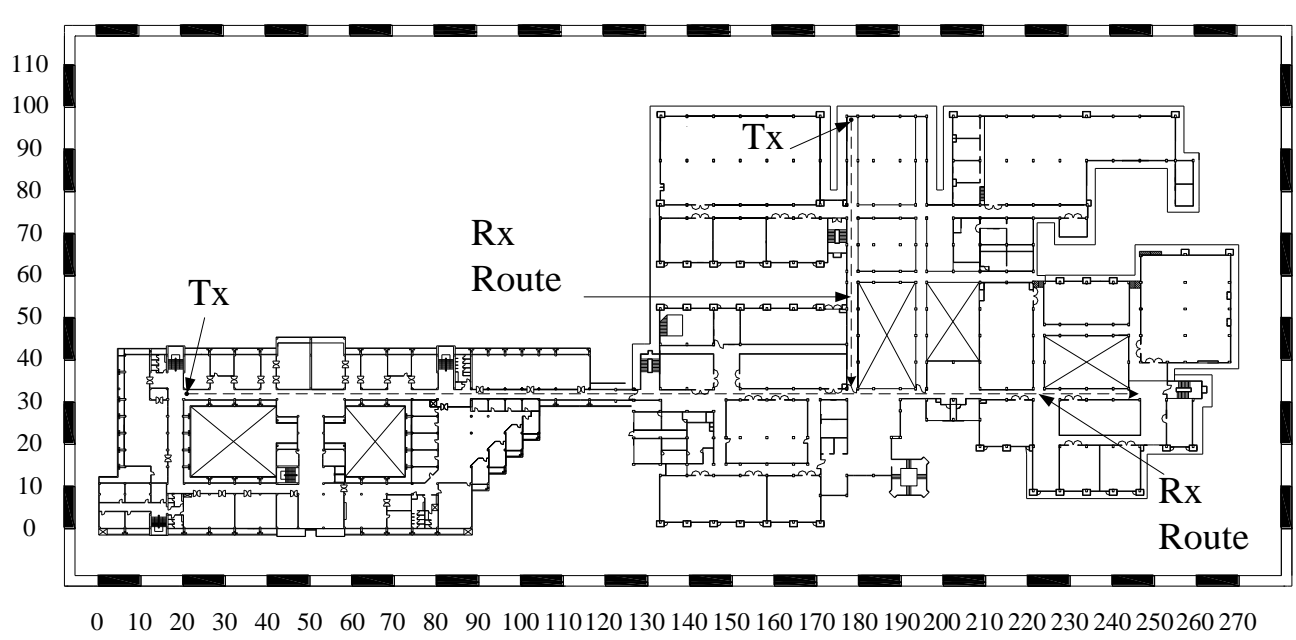

Fig. 2: Measurement location of the concrete school building.

\subsubsection{The future car park building}

The parking building of future park center was completed in 1990. It consists of eight floors with dimensions of $130 \times 270 \mathrm{~m}^{2}$ as shown in Fig. 3. The construction of the car park building is of concrete column and flat ceiling, the floor to ceiling height is $2.25 \mathrm{~m}$. The ceilings are mostly hard concrete. The building capacity is about 300 cars per floor. 


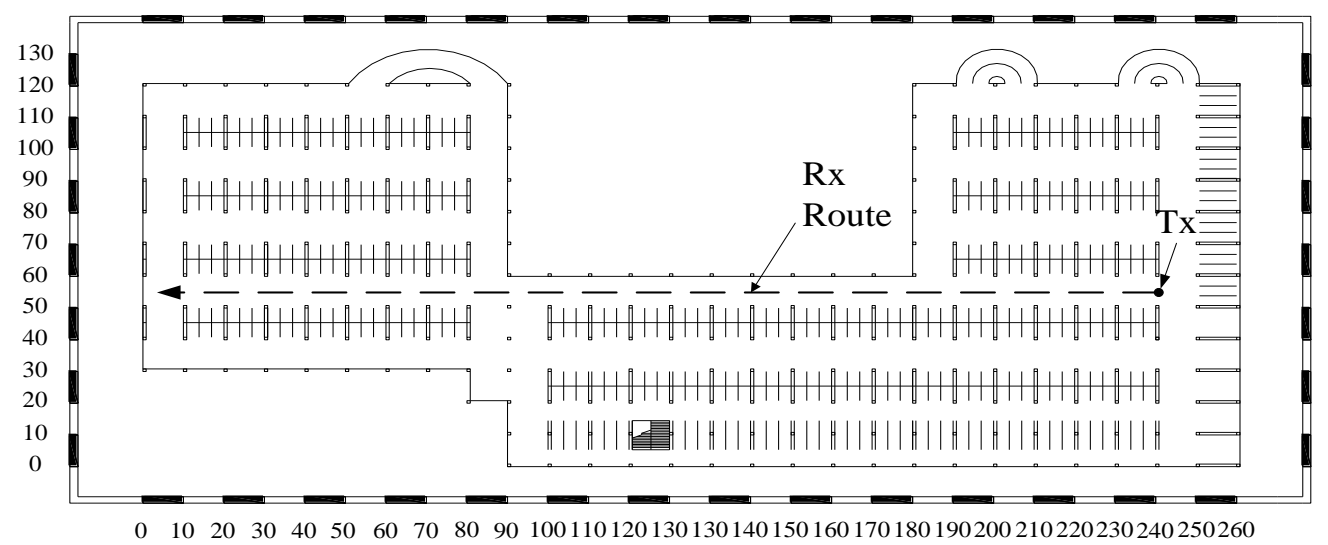

Fig. 3: Measurement location of the Future park building.

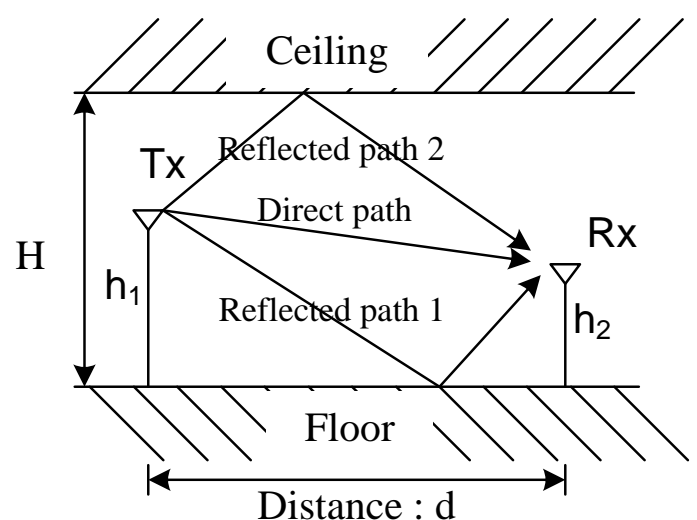

Fig. 4: Three-ray model.

\section{SINGLE REGRESSION LINE MODELS}

The mean LOS path loss is a function of distance to the $n$ power as below

$$
L_{\text {LOS }}=L\left(d_{0}\right)+10 \log _{10}\left(\frac{d}{d_{0}}\right)^{n}
$$

where $L_{L O S}$ is the path loss at distance d from the transmitter and $L\left(d_{0}\right)$ is the path loss at the reference distance, $d_{0}$ from the transmitter. Because of first Fresnel zone region, propagation loss as a function of distance has two distinct regions as follow

$$
P L_{b p}(d)= \begin{cases}L_{b p, 1}+10 \log _{10}\left(\frac{d}{d_{b p}}\right)^{n_{1}}, & \text { for } d \leq d_{b p} \\ L_{b p, 2}+10 \log _{10}\left(\frac{d}{d_{b p}}\right)^{n_{2}}, & \text { for } d>d_{b p}\end{cases}
$$

Where $d_{b p}$ is the distance of the breakpoint from the transmitter, $L_{d b, 1}$ and $L_{d b, 2}$ are path loss at 410 
breakpoint distance on either side of the breakpoint, and $n_{1}$ and $n_{2}$ are the path-loss exponents on either side of the breakpoint.

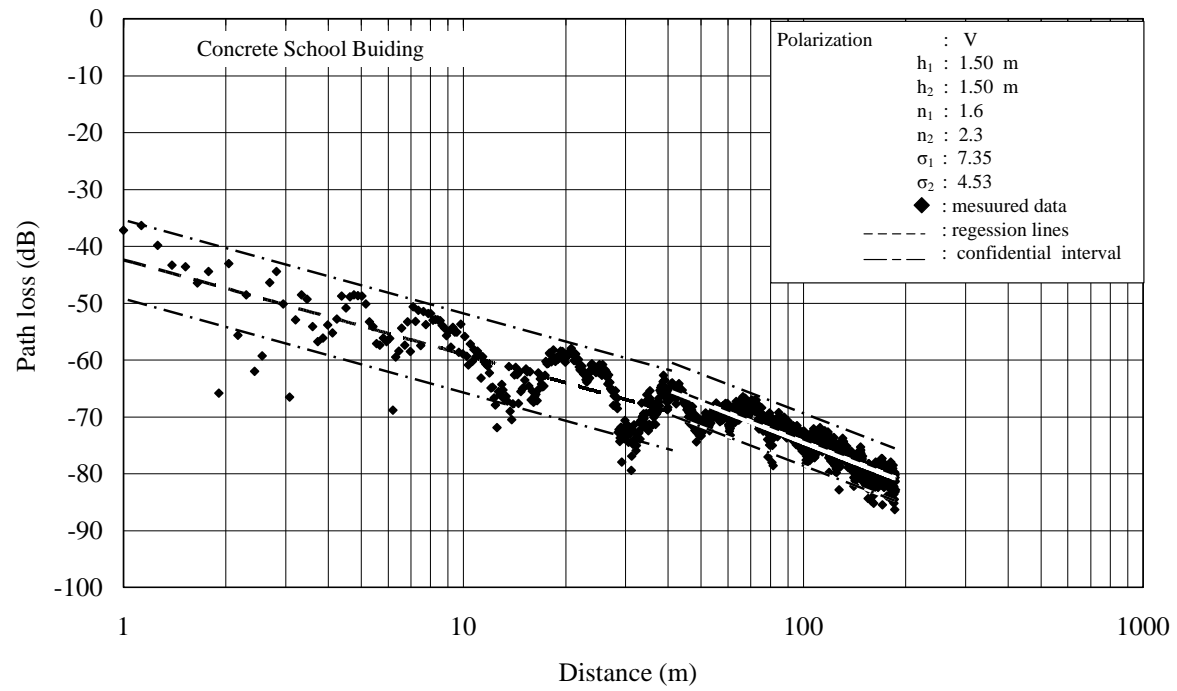

a) Second floor (no people moving)

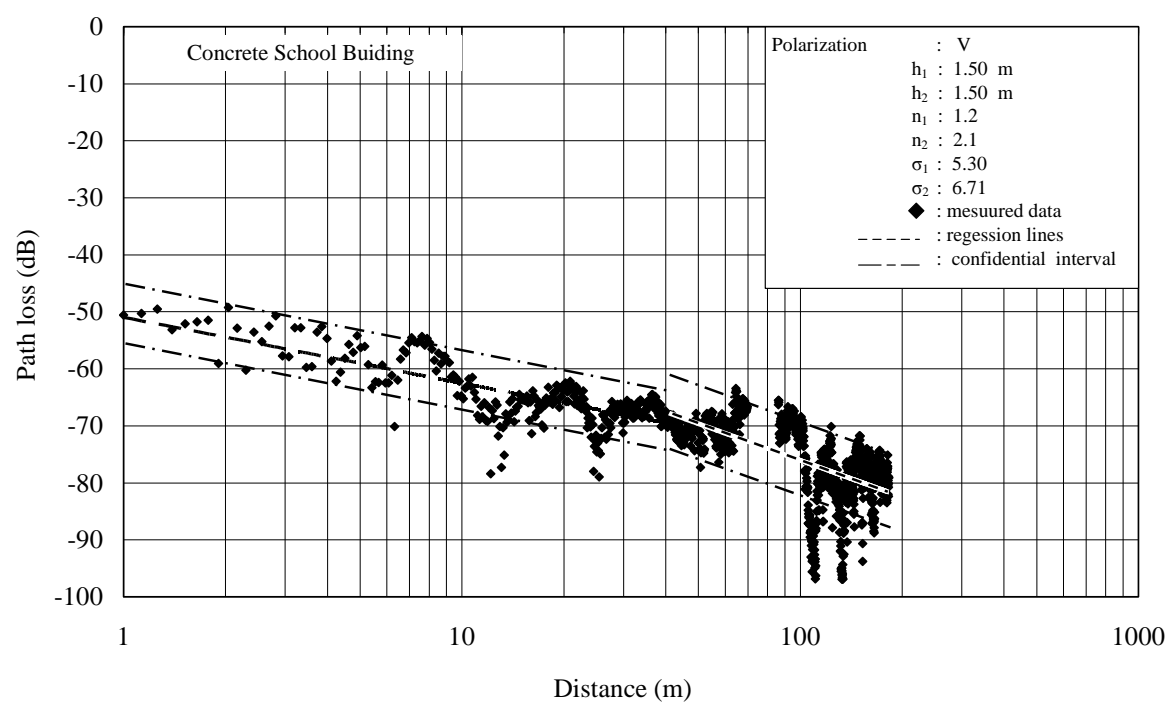

b) First floor (with people moving)

Fig. 5: Shows path loss measurement and the regression lines in concrete school building.

The breakpoint distance $d_{b p}$, can be calculated by using the diameter of the first Fresnel zone, a distance $d$ from the transmitter in a corridor, is approximately given by $\mathrm{Z}_{\mathrm{f}} \approx \sqrt{\lambda d}$. However, for the large corridor which has a width much more than ceiling height or where the reflections from side walls suffer from numerous losses due to penetration through glass, porous materials, etc., before reflection, we found that the first Fresnel zone effect then depended on only reflecting from ceiling and floor and was calculated by using a three-ray model [10] in Fig. 4 from

$$
d_{b p}=4\left(H-h_{2}\right) \mathrm{h}_{2} / \lambda
$$


where $\mathrm{H}$ and $\mathrm{h}_{2}$ are the ceiling height and receiving antenna height respectively. The path lossdistance characteristics of the concrete school building are shown in Figure 5. a-c. We found that the breakpoint distance in Figure 5 a) was the same one in Figure 5 b). This confirms that there are only three dominant ray paths travelling from the transmitter to the receiver. Figure 5 c) shows that there are a lot of fast fading and path loss exponents changed when people move between the transmitter and the receiver. It is observed that the disconnected scatter plots are caused by two sub-building separations on the first floor.

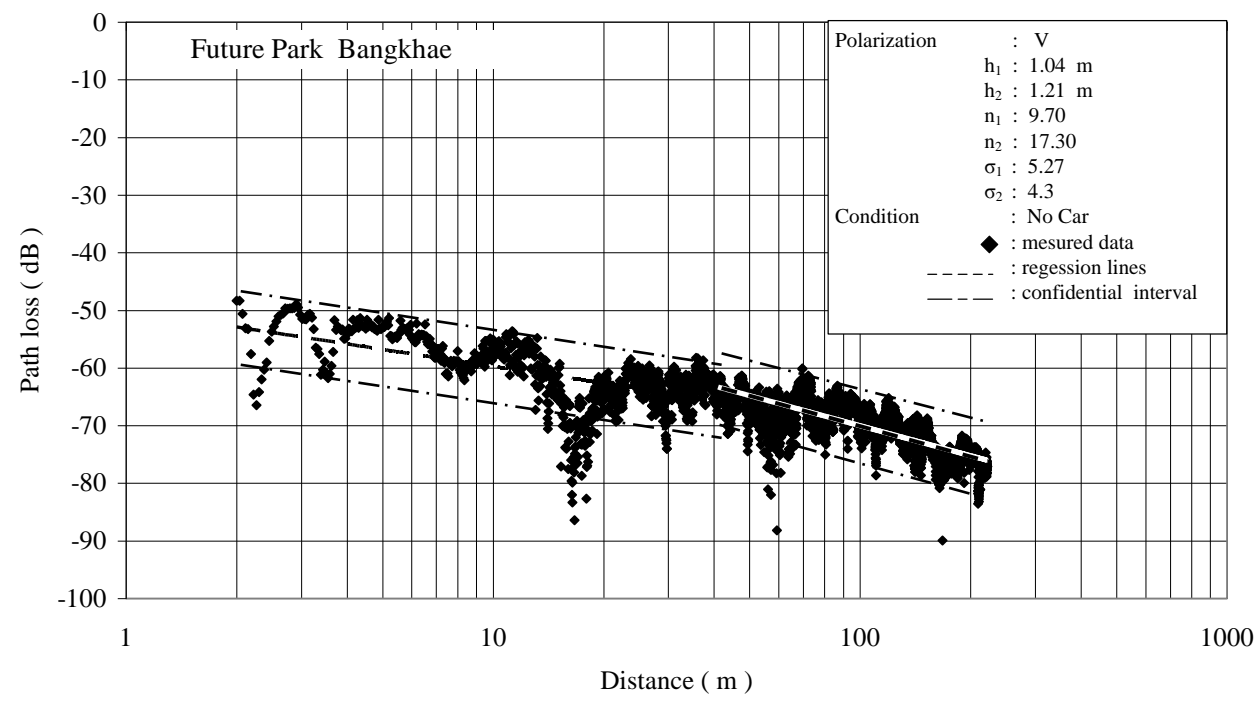

a) no cars moving

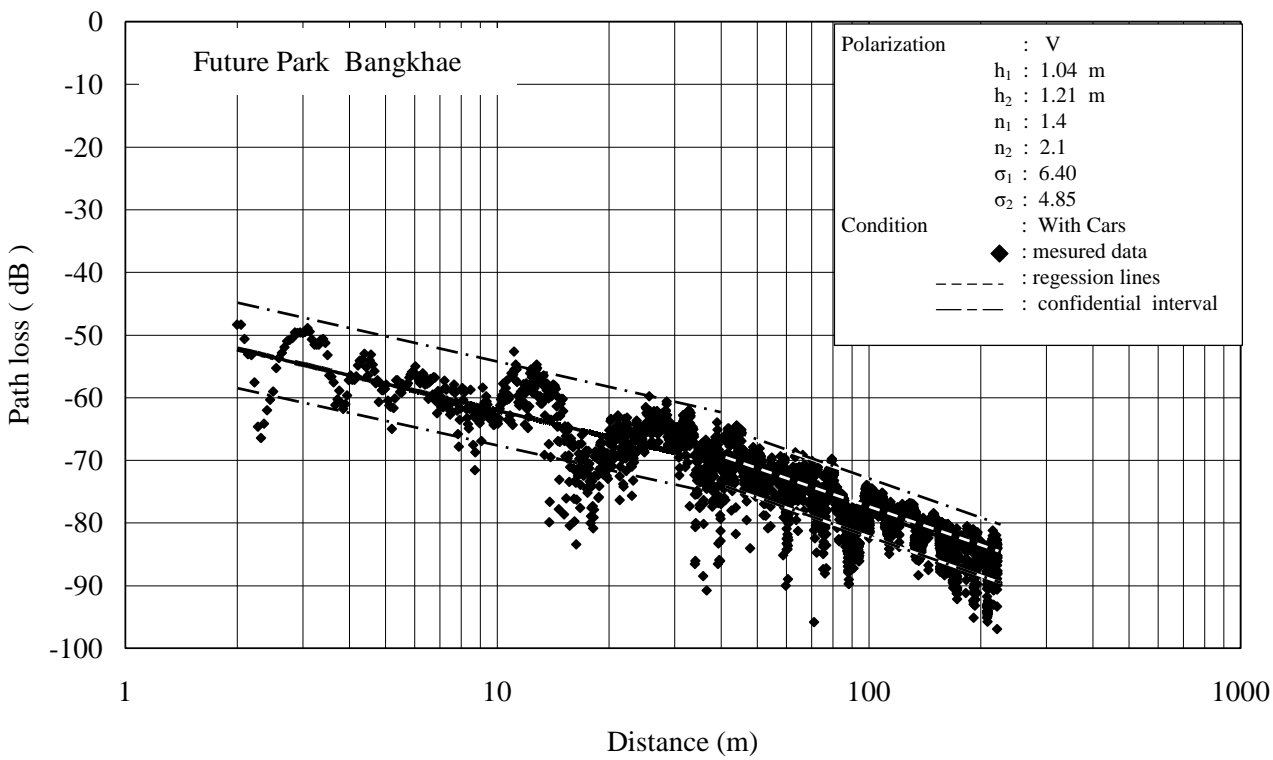

b) with cars moving

Fig. 6: Shows path loss measurement and the regression lines in Future park building.

Figure 6 shows scatter plot and regression line of path loss-distance characteristics in the Future Park building. We also found that there are a lot of fast fading and path loss exponent changed 
when cars move between the transmitter and the receiver. The dot lines in the figures show the regression lines before and after breakpoints. To recheck the breakpoint distance, we used the breakpoint discrimination method defined as

$$
r(d)=\frac{\sum_{d>}\left(y_{i}-Y_{1 i}\right)^{2}}{N_{1}}+\frac{\sum_{d<}\left(y_{i}-Y_{2 i}\right)^{2}}{N_{2}}
$$

where $y_{i}, Y_{1 i}$ and $Y_{2 i}$ are measured data regression line before and after breakpoint respectively. $\mathrm{N}_{1}$ and $\mathrm{N}_{2}$ are the numbers of data before and after breakpoint respectively.

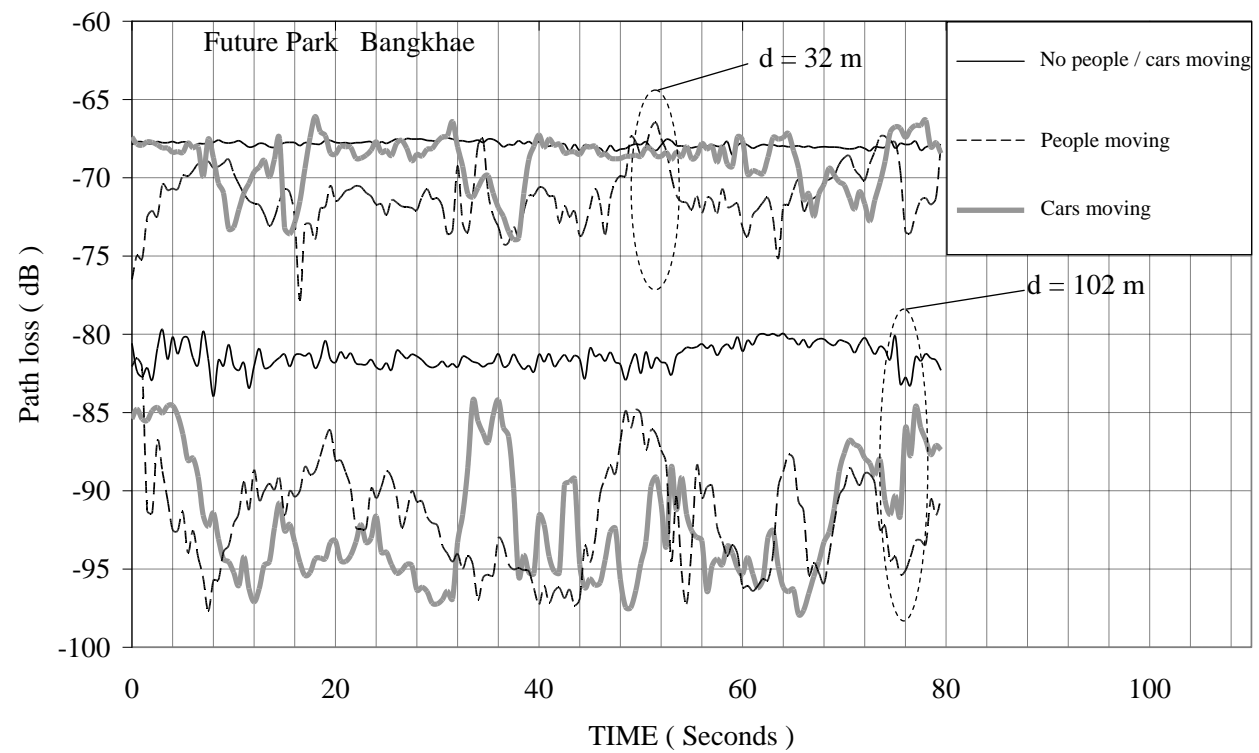

Fig. 7: Shows fading path loss measurement.

Figure 7 shows the time-variant effects to path loss fading at distance of 32 and $102 \mathrm{~m}$ from the transmitter. We found that there are small differences in fading between people and cars moving at the same measurement point and larger fading at distance far from the breakpoint.

The estimation for the path loss exponents $n$ and coefficients of determination $R^{2}$ regressing by one straight line are summarized in Table I, where subscript 1 and 2 denote the case for before and after breakpoint distances respectively.

Table 1: Estimation of path loss exponent and coefficients of determination.

\begin{tabular}{lccccccccc}
\hline Building Type & \multicolumn{3}{c}{ no time-variant effect } & \multicolumn{5}{c}{ with time-variant effect } \\
\hline & $n_{1}$ & $n_{2}$ & $R^{2}{ }_{l}$ & $R^{2}{ }_{2}$ & $n_{1}$ & $n_{2}$ & $R^{2}{ }_{I}$ & $R^{2}{ }_{2}$ \\
Concrete school & & & & & & & & \\
$\quad$ - First floor & 1.3 & 1.7 & 0.68 & 0.72 & 1.2 & 2.1 & 0.67 & 0.37 \\
$\quad$ - Second floor & 1.6 & 2.6 & 0.69 & 0.83 & - & - & - & - \\
Future Park & 1.0 & 1.7 & 0.52 & 0.7 & 1.4 & 2.1 & 0.40 & 0.58 \\
\hline
\end{tabular}


The path loss exponent, $n_{1}$, was less than 2.0 and $n_{2}$, was less than 4.0. This is because of the influence of wave-guiding effects in the first Fresnel zone region. The coefficient of determination, $R^{2}$, is an index of how good the regression is. The value of $R^{2}$ for regression with people or cars was always less than that with no people or no cars. This is because there was fast fading from people or cars shadowing making the path loss data scatter from the regression line. The confident intervals as shown in Figures 5 and 6 provide prediction accuracy within a certain range of standard deviation. However the fast fading data was still out of the line intervals

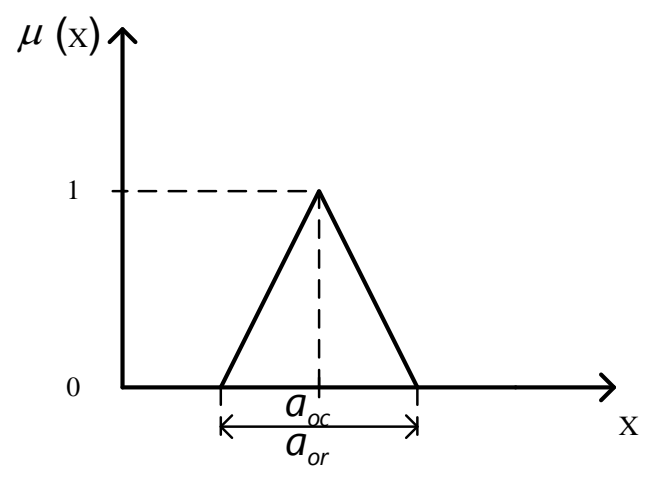

Fig. 8: Triangular from of fuzzy number.

\section{NEW UPPER AND LOWER BOUND MODELS}

Upper and lower bound evaluations for linear regression analysis have been done using fuzzy liner regression model (FLR model) [11]. We applied those evaluations to the mobile propagation path loss characteristics as follows:

The general regression model is written in the from

$$
\gamma=Z \widetilde{A}
$$

where:

$$
y_{i}\left(z_{l}\right)=\tilde{a}_{0}+\tilde{a}_{1} z_{i 1}+\ldots .+\tilde{a}_{k} z_{i k} \quad \mathrm{I}=1,2, \ldots, \mathrm{n}
$$

The FLR model is represented using symmetric triangular fuzzy parameters $\tilde{a}_{i=}\left[a_{i c}, a_{i r}\right]$ as shown in figure 8 by:

$$
\begin{gathered}
y_{i}\left(z_{i}\right)=\left[a_{0 c}, a_{0 r}\right]+\left[a_{1 c}, a_{1 r}\right] z_{i l}+\ldots .+\left[a_{k c}, a_{k r}\right] z_{i k} \\
y_{i c}\left(z_{i}\right)=a_{0 c}+a_{1 c} z_{i l}+\ldots .+a_{k c} z_{i k} \\
y_{i r}\left(z_{i}\right)=a_{0 r}+a_{1 r} z_{i l}+\ldots .+a_{k r} z_{i k}
\end{gathered}
$$

where: $y_{c}, a_{c}$ are center parameters of fuzzy numbers at membership function $\mu=1, y_{r}, a_{r}$ are spreads of fuzzy numbers, geometrically the spread is a half of the base of the triangular.

The parameters $\tilde{a}_{i}$ of the vector $\tilde{A}$ of the FLR model are determined by a solution of a linear programming, LP problem which is to minimize the sum of spreads $y_{r}\left(z_{i}\right)$ of elements of vector y Therefore the following LP problem is formulated.

$$
C=y_{i r}(z)+y_{2 r}\left(z_{2}\right)+\ldots y_{n r}\left(z_{n}\right) \longrightarrow \text { Minimum }
$$

Subject to

$$
y_{i} \in Y\left(z_{i}\right), \quad \mathrm{i}=1,2, \ldots, \mathrm{n}
$$




$$
a_{i r} \geq 0, \quad \mathrm{i}=0,1,2, \ldots, \mathrm{k}
$$

from the FLR model, the LP problem then can be written as follows:

$$
\begin{gathered}
\sum_{i=1}^{n}\left(a_{o r}+a_{1 r}\left|z_{i l}\right|+\ldots+a_{k r}\left|z_{i k}\right|\right) \longrightarrow \text { Minimum } \\
a_{0 c}+\sum_{j=1}^{k}\left(a_{j c} a_{i j}\right)-a_{0 r}-\sum_{j=1}^{k}\left(a_{j r} \mid z_{i j}\right) \leq y_{i}, i=1,2, \ldots, n \\
a_{0 c}+\sum_{j=1}^{k}\left(a_{j c} a_{i j}\right)+a_{0 r}+\sum_{j=1}^{k}\left(a_{j r} \mid z_{i j}\right) \geq y_{i}, i=1,2, \ldots, n
\end{gathered}
$$

The parameters $a_{i}=\left[a_{i c}, a_{i r}\right]$ of vector $\tilde{A}$ are determined as the optimal solution of the LP problem. The FLR models for propagation path loss are presented in form

$$
L_{\text {LOS }}=\left[a_{0 c}, a_{0 r}\right]+\left[a_{1 c}, a_{1 r}\right] \log (d)
$$

Where upper bound can be written as

$$
L_{\text {LOS }, u}=\left[a_{0 c}+a_{0 r}\right]+\left[a_{1 c}+a_{1 r}\right] \log (d)
$$

and lower bound can be written as

$$
L_{\text {LOS }, 1}=\left[a_{0 c}-a_{0 r}\right]+\left[a_{1 c}-a_{1 r}\right] \log (d)
$$

Where $\mathrm{d}=$ distance between transmitter and receiver. The LP problem corresponding to the given data was formulated. By solving this LP problem, the upper and lower bound path loss models for the two buildings are obtained as follow:

\subsection{Concrete school building}

$$
\begin{aligned}
L_{L O S} & =[49.6,11.5]+[15.4,0] \log (d) ; \text { for } d \leq d_{b p} \\
L_{L O S} & =[10.8,0]+[35.5,7.1] \log (d) ; \text { for } d>d_{b p}
\end{aligned}
$$

\subsection{Future park building}

$$
\begin{aligned}
L_{L O S} & =[44.5,10.8]+[12.3,0] \log (d) ; \text { for } d \leq d_{b p} \\
L_{L O S} & =[27.5,2.5]+[28.5,6.5] \log (d) ; \text { for } d>d_{b p}
\end{aligned}
$$

From above results, the average path loss exponents $\mathrm{n} 1$ and $\mathrm{n} 2$ were $(15.4+12.3) / 2=13.9$ and $((35.5-7.1)+(28.5-6.5)) / 2=25.2$. Since the maximum fading depth indoor propagation was about $30 \mathrm{~dB}$, we modified the boundary indoor path loss models as

$$
L_{L O S, u}=L_{b p}+ \begin{cases}14 \log _{10}\left(\frac{d}{d_{b p}}\right), & \text { for } d \leq d_{b p} \\ 25 \log _{10}\left(\frac{d}{d_{b p}}\right), & \text { for } d>d_{b p}\end{cases}
$$

and 


$$
L_{L O S, l}=L_{b p}+30+ \begin{cases}14 \log _{10}\left(\frac{d}{d_{b p}}\right), & \text { for } d \leq d_{b p} \\ 25 \log _{10}\left(\frac{d}{d_{b p}}\right), & \text { for } d>d_{b p}\end{cases}
$$

when $L_{b p}$ is propagation loss at $d_{b p}$. According to the three-ray model in Fig. 4, the received power $\operatorname{Pr}$ is

$$
P_{r}=P_{t}\left(\frac{\lambda}{2 \pi d}\right)^{n} \sin ^{2} \frac{\Delta}{2}
$$

Where Pt is the transmitted power, and $\Delta$ the phase difference between the direct and reflected waves. A lower bound occurs when $\Delta / 2=\pi / 2$. By using $d_{b p}=4\left(H-h_{2}\right) \mathrm{h}_{2} / \lambda$ and above equation, setting $d=d_{b p}$, and $\sin ^{2}(\Delta / 2)=1, \mathrm{~L}_{\mathrm{bp}}$ can be defined as

$$
L_{b p}=\left|20 \log _{10}\left\{\frac{\lambda^{2}}{8 \pi\left(H-h_{2}\right) h_{2}}\right\}\right|
$$

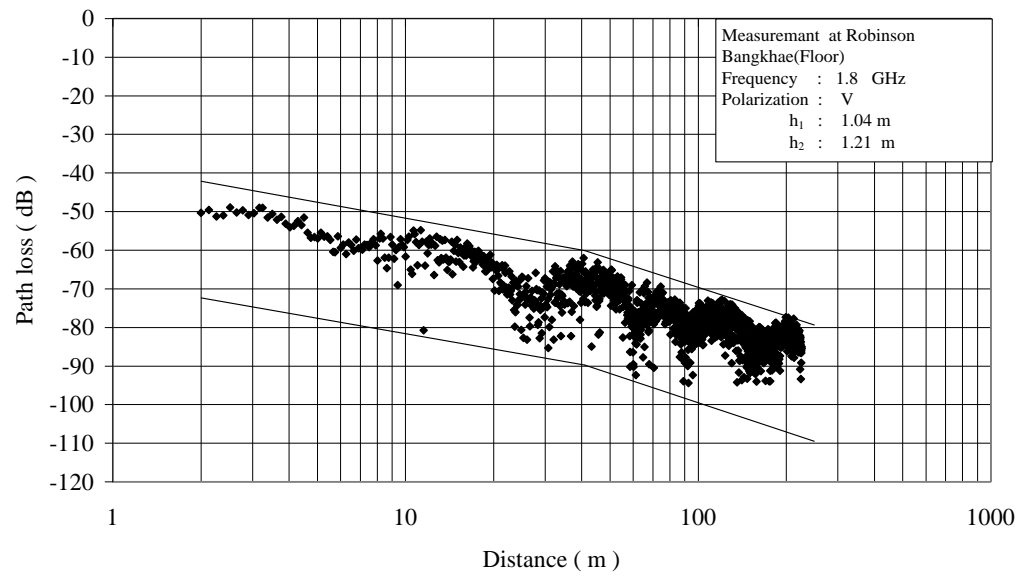

a) Future park building

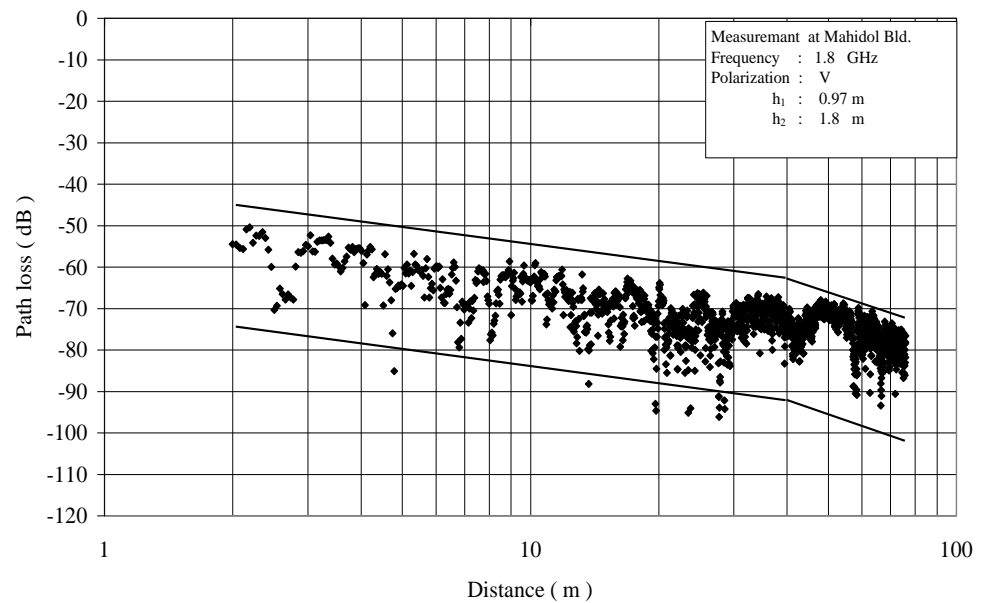

b) Concrete school building

Fig. 9: Shows path loss measurement and the proposed models in different building. 
To validate the proposed model, we performed path loss measurements for different locations at high traffic hours in the buildings. Comparisons between proposed models and measured path loss are shown in Figure 9. Estimated path loss bounds are shown by the solid lines in the figure. The upper and lower bound models agree with the measured data at the Future Park building with ceiling height of $2.25 \mathrm{~m}$. and the Mahidol school building with ceiling height of $2.77 \mathrm{~m}$. as shown in Figure $9 \mathrm{a}$ )-b) respectively. Note that the value of distance breakpoints are varied with the transmitting and receiving antennas in order that the path loss data after the break points are exited within the LOS path.

\section{CONCLUSION}

New upper and lower bound models were proposed for mobile communication in the business building. These models were based on fuzzy linear regression of the measured data. The path loss measurements were performed at a frequency of $1.8 \mathrm{GHz}$ in LOS corridor of the buildings. We found that the breakpoint characteristics influenced from ceiling height and antenna height. These models agree with the measured data in mobile communication band.

The boundary model predicts path loss accuracy within the boundary that will be useful for the system and cell design of indoor wireless communication systems in the realistic propagation environments including for limiting of the received signal level in order to comply with public health regulations.

\section{ACKNOWLEDGEMENT}

This work was supported by a medium research grant (Round 6/2004) from Mahidol University, Thailand.

\section{REFERENCES}

1. Seidel, S.Y. and Rappaport, T.S. (1992), $914 \mathrm{MHz}$ path loss prediction models for indoor wireless communications in multifloored buildings, IEEE Trans. Antennas and Propagat., vol. 40, No. 2, pp. 207-217.

2. Jarmo Kivinen, Xiongwen Zhao, and Pertti Vainikainen (2001), Empirical Characterization of Wideband Indoor Radio Channel at 5.3 GHz," IEEE Trans. Antennas and Propagat., vol. 49, No. 8, pp. 1192-1203.

3. Tarng, J.H., Chang, W.R., and Hsu, B.J. (1997), Three-dimensional modelling of 900-MHz and 2.44-GHz radio propagation in corridors" IEEE Trans. Vehicular Tech., vol. 46, No. 2.

4. Remley, K.A., Anderson, H.R. and Weisshar, A. (2000), Improving the accuracy of Raytracing Techniques for Indoor Propagation Modeling" IEEE Trans. Vehicular Tech., vol. 49, No. 6.

5. Honcharenko, W., Bertoni, H.L., Dailing, J.L., Qian, J., and Yee, H.D. (1992), Mechanisms governing UHF propagation on single floors in modern office building, IEEE Trans. Vech.Technol., vol. 41, No. 4, pp. 496-504.

6. Cheung, K.W., Sau, J.H.-M., and Murch, R.D. (1998), A New Empirical Model for Indoor Propagation Prediction IEEE Trans. Vech.Technol., vol. 47, No. 3, pp. 996-1001.

7. Ghaddar, M., Talbi, L., and Denidni, T.A. (2004), Human body modelling for prediction of effect of people on indoor propagation channel, Electronics letters, vol. 40, No. 25. 
8. Milstein, L.B., Schilling, D.L., Pickholtz, R.L., RCEG, V.E., Kullback, M., Kanterrakis, E.G., Fishman, D.S., Biederman, W.H., and Ssalerno, D.C. (1992), On the feasibility of a CDMA overlay for personal communications network, services" IEEE J. Select. Areas Commun., vol.10, pp. 655-668.

9. Masui, H., Kobayashi, T., and Akaike, M. (2002), Micro-wave path loss modeling in urban line-of-sight. environments", IEEE J. Select. Areas Commun., vol. 20, pp. 1151-1155.

10. Saleh, F. (1996), Cellular Mobile Systems Engineering," Artech House Publishers Boston London.

11. Tanaka, H., Uejima, S., and Asai, K. (1982), Linear regression analysis with fuzzy model," IEEE Trans. Syst.,Man.,Cybern., vol. SMC-12, pp. 903-907. 